\title{
Magnetic Field Tuning and Quantum Interference in a Cooper Pair Splitter
}

\author{
G. Fülöp, ${ }^{1}$ F. Domínguez, ${ }^{2}$ S. d'Hollosy, ${ }^{3}$ A. Baumgartner, ${ }^{3, *}$ P. Makk, ${ }^{1,3}$ M. H. Madsen, ${ }^{4, \dagger}$ V. A. Guzenko, ${ }^{5}$ \\ J. Nygård, ${ }^{4}$ C. Schönenberger, ${ }^{3}$ A. Levy Yeyati, ${ }^{2}$ and S. Csonka ${ }^{1}$ \\ ${ }^{1}$ Department of Physics, Budapest University of Technology and Economics, \\ and Condensed Matter Research Group of the Hungarian Academy of Sciences, \\ Budafoki út 8, 1111 Budapest, Hungary \\ ${ }^{2}$ Departamento de Física Teórica de la Materia Condensada, Condensed Matter Physics Center (IFIMAC), \\ and Instituto Nicolás Cabrera, Universidad Autónoma de Madrid, E-28049 Madrid, Spain \\ ${ }^{3}$ Department of Physics, University of Basel, Klingelbergstrasse 82, CH-4056 Basel, Switzerland \\ ${ }^{4}$ Center for Quantum Devices \& Nano-Science Center, Niels Bohr Institute, University of Copenhagen, \\ Universitetsparken 5, DK-2100 Copenhagen, Denmark \\ ${ }^{5}$ Laboratory for Micro- and Nanotechnology, Paul Scherrer Institute, CH-5232 Villigen PSI, Switzerland
}

(Received 3 July 2015; published 25 November 2015)

\begin{abstract}
Cooper pair splitting (CPS) is a process in which the electrons of the naturally occurring spin-singlet pairs in a superconductor are spatially separated using two quantum dots. Here, we investigate the evolution of the conductance correlations in an InAs CPS device in the presence of an external magnetic field. In our experiments the gate dependence of the signal that depends on both quantum dots continuously evolves from a slightly asymmetric Lorentzian to a strongly asymmetric Fano-type resonance with increasing field. These experiments can be understood in a simple three-site model, which shows that the nonlocal CPS leads to symmetric line shapes, while the local transport processes can exhibit an asymmetric shape due to quantum interference. These findings demonstrate that the electrons from a Cooper pair splitter can propagate coherently after their emission from the superconductor and how a magnetic field can be used to optimize the performance of a CPS device. In addition, the model calculations suggest that the estimate of the CPS efficiency in the experiments is a lower bound for the actual efficiency.
\end{abstract}

DOI: 10.1103/PhysRevLett.115.227003

PACS numbers: 74.45.+c, 03.67.Bg, 73.23.-b, 73.63.Nm

In the Cooper pair splitting (CPS) process the electrons of the Cooper pairs in a superconductor are separated spatially using two quantum dots (QDs) coupled in parallel to a central superconductor contact $(S)$ in a three-terminal geometry [1-3], see Fig. 1(a). The Coulomb repulsion on the QDs and the quasiparticle energy gap of the superconductor enforce the electrons to separate into different normal metal electrodes ( $N 1$ and $N 2$ ). Since Cooper pairs are spin-singlet states, such devices could serve as a source of nonlocal spin entangled electron pairs. Similar geometries are also relevant in the search for Majorana bound states [4] in local $S-N$ junction experiments and in threeterminal devices, where an increase in CPS efficiency might serve as a signature of the elusive exotic states [5].

In a series of recent experiments on semiconducting nanowires (NWs) [6-9], carbon nanotubes [10-12], and graphene [13], CPS was demonstrated by positive conductance correlations between the currents from $S$ into $N 1$ and $N 2$. In these experiments, external magnetic fields were solely used to suppress the superconductivity for control experiments, but not as a parameter to tune CPS. In addition, most experiments were interpreted in terms of an incoherent picture with independent transport mechanisms, only coupled by the QD dynamics $[9,11]$.

Here, we report experiments in a NW-based Cooper pair splitter with a $\mathrm{Nb}$ superconducting electrode. The large critical magnetic field of $\mathrm{Nb}$ allows us to explore CPS up to $\sim 1 \mathrm{~T}$. We find that the conductance correlations can not only manifest as symmetric peaks and dips in the gate dependence, but also as strongly asymmetric shapes reminiscent of Fano resonances. We interpret the experimental results in a minimal model that incorporates the superconducting proximity effect, the tunnel coupling between the QDs, and quantum interference [see Fig. 1(b)]. Interference results in asymmetric Fano-type features for

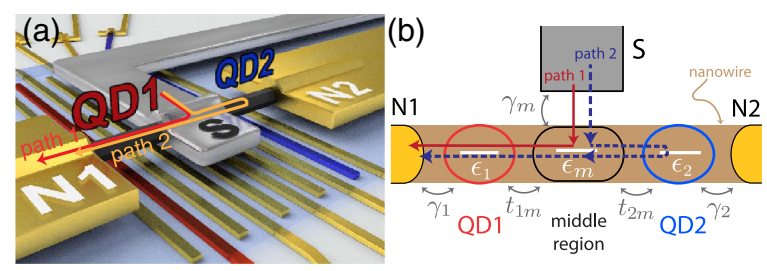

FIG. 1 (color online). (a),(b) Schematics of a Cooper pair splitter and the corresponding three-site model in which $S$ is coupled to the QDs by a central NW region. The dashed and solid lines represent two different interfering single-electron paths from $S$ to $N 1$, which can result in Fano-type conductance resonances. $\epsilon_{1,2}$ are the QD energy levels, $\epsilon_{m}$ is the level position of the middle region, $\gamma_{1, m, 2}$ are the tunnel coupling strengths to the respective reservoirs, and $t_{1 m, 2 m}$ are the couplings between the middle region and the QDs. 
local transport processes, whereas CPS produces Lorentzian shaped contributions to the conductances. These effects can be strong enough to obscure the positive correlations due to CPS. The interference features in our experiments suggest that electrons can be transmitted coherently through a CPS device, which is a fundamental prerequisite for testing Bell's inequality in a beam mixer setup [14]. Since often the spin-coherence time is longer than the spatial coherence time, our results are also encouraging for the various propositions to detect spin entanglement [15-19].

Figure 1(a) shows a schematic of a CPS device. Using electron-beam lithography, an array of twelve $40 \mathrm{~nm}$ wide local bottom gates (4/18 nm Ti/Pt, $\sim 60 \mathrm{~nm}$ spacing) was fabricated on a silicon substrate, electrically insulated by $\sim 25 \mathrm{~nm} \operatorname{SiN}_{x}$. A single NW ( 70 nm diameter) was then deposited perpendicular to the gates using micromanipulators. The NWs were grown by solid-source molecular beam epitaxy [20], using an optimized process to suppress stacking faults [21]. A $330 \mathrm{~nm}$ wide and $110 \mathrm{~nm}$ thick superconducting $\mathrm{Nb}$ contact in the center and two normal metal electrodes (7/95 nm Ti/Au) at the ends of the NW were fabricated consecutively by conventional electron beam lithography, using an ammonium sulfide passivation [22] to remove the native NW oxide.

The experiments were carried out in a dilution refrigerator at a base temperature of $\sim 50 \mathrm{mK}$. The QDs on either side of $S$ were each induced in the NW by a negative voltage applied to two neighboring bottom gates, of which one was also used to tune the chemical potential of the respective QD. We label the voltages on these two tuning gates as $V_{g 1}$ and $V_{g 2}$ and use the terms "local gate" and "far gate" to distinguish the two gates when discussing a specific QD. A sinusoidal voltage $V^{(\mathrm{ac})} \approx 10 \mu \mathrm{V}$ applied to $S$ results in the simultaneously recorded currents $I_{1}^{(\mathrm{ac})}$ and $I_{2}^{(\mathrm{ac})}$ in the contacts $N 1$ and $N 2$, which were held at carefully nulled potentials. We define the differential conductance through each QD as $G_{i}=I_{i}^{(\mathrm{ac})} / V^{(\mathrm{ac})}$, both of which show well-defined, uncorrelated Coulomb blockade diamonds [23].

Figure 2(a) shows $G_{1}$ and $G_{2}$ as a function of the two QD tuning gates for $B=0$. The resonance amplitudes of both QDs are increased when both are on resonance (resonance crossing), which results in a positive correlation between the conductance variations. In contrast, in the measurements at $B=1 \mathrm{~T}$ in Fig. 2(b), the conductance maxima do not occur exactly at the expected resonance crossings, but vary in position, which results in a conductance not symmetric with respect to a crossing. Figures 2(c) and 2(d) show cross sections taken on top of the QD resonance as a function of the respective far gate for a series of magnetic fields. Away from a resonance crossing the resonance positions and amplitudes are independent of the respective far gate, while near a crossing both vary with
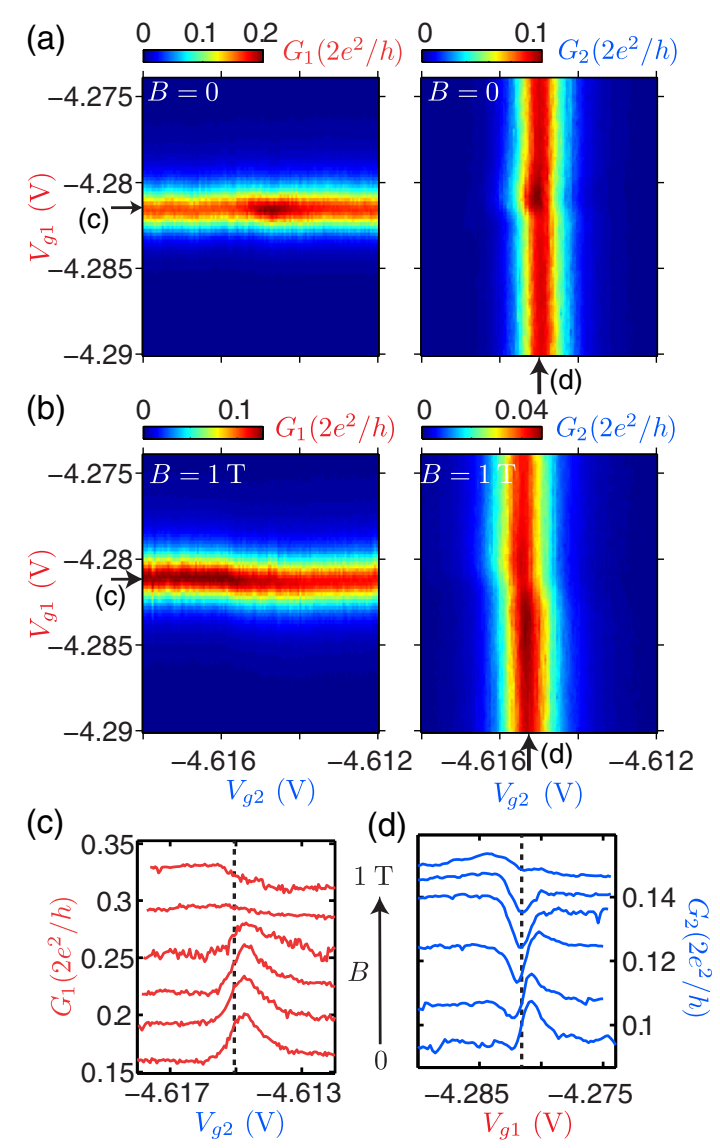

FIG. 2 (color online). (a),(b) $G_{1}$ and $G_{2}$ as a function of $V_{g 1}$ and $V_{g 2}$ for $B=0$ and $B=1 \mathrm{~T}$, respectively. (c),(d) $G_{1}$ and $G_{2}$ as a function of the respective far gate $V_{g 2}$ and $V_{g 1}$ for a series of magnetic fields $B$ with the local gates set to a QD resonance, as indicated by black arrows in (a) and (b). The dashed lines indicate the resonance crossings. All curves are shifted vertically for clarity.

gate voltage and exhibit different symmetries with respect to the resonance crossing (dashed lines) with increasing $B$. For $B=0$ both resonance modulations are dominated by a roughly Lorentzian shape with a small minimum at the lowvoltage side of the resonance crossing. As we increase the magnetic field, $G_{1}$ and $G_{2}$ evolve differently: while $G_{1}$ changes only little up to $B \approx 400 \mathrm{mT}$ and then becomes broadened at higher fields, the variation of $G_{2}$ first evolves into a dip-peak structure, then into a dip at intermediate fields and into a maximum at the low-voltage side of the resonance crossing for the highest fields. The finite-field curves are reminiscent of Fano-type resonances, rather than Lorentzians. Directly comparing the $B=0$ to the $B=1 \mathrm{~T}$ curve, one finds that the conductance maximum is shifted from the right to the left side of a crossing. We note that for most curves the maxima and minima in the conductance variations do not coincide with the resonance crossing.

The resemblance of the experimental conductance variations to Fano-type resonances suggests that at least one of the transport mechanisms is prone to quantum interference, which cannot be described in the incoherent models of 
previous works $[9,11]$. To account for the observed interference patterns also requires us to go beyond earlier coherent two-dot models [10,25,26], where the QDs are coupled by direct tunneling. Here, we introduce a three-site model, shown schematically in Fig. 1(b), in which each QD is represented by a single spin-degenerate level with Coulomb interactions and coupled coherently to a central NW segment below $S$ and to the leads $N 1$ and $N 2$. The central segment is also modeled as a single level coherently coupled to $S$. The Hamiltonian (without the coupling to the leads [23]) reads

$$
H_{0}=\sum_{\sigma, i} \epsilon_{i, \sigma} n_{i, \sigma}+U_{i} n_{i, \uparrow} n_{i, \downarrow}+\sum_{\sigma, i \neq m} t_{i m} d_{i, \sigma}^{\dagger} d_{m, \sigma}+\text { H.c. }
$$

where $i=1, m, 2$ label the left, middle, and right levels with the on-site energies $\epsilon_{i, \sigma}=\epsilon_{i}(B)+\sigma g_{i} B / 2$, comprising orbital and Zeeman shifts. For the $g$ factors $g_{i}$ we use typical values in the range of $|g|=5-15$ [27,28]. $t_{i m}$ corresponds to the hopping amplitudes from the QDs to the central site. To simulate the superconducting proximity effect we set the Coulomb interactions on the central region to zero, $U_{m}=0$, which is justified by the large size and the screening by the superconductor. This assumption allows the central level to be occupied by two electrons, e.g., by a Cooper pair from $S$. We assume that only this central region is coupled to $S . \gamma_{i}$, with $i=1, m, 2$, are the tunneling rates from the three sites to the respective lead, see Fig. 1(b). We calculate the linear transport characteristics using an equation of motion approach for the electronic Green's functions $[23,29]$. The conductances can be decomposed as $G_{i}=G_{\mathrm{loc} ; i}+G_{\mathrm{CPS}}$ where the local contributions comprise local Andreev reflection (LAR) and single quasiparticle tunneling, $G_{\mathrm{loc} ; i}=$ $G_{\mathrm{LAR} ; i}+G_{\mathrm{qp} ; i} \cdot G_{\mathrm{CPS}}$ denotes the contribution due to CPS.

The three-site model qualitatively reproduces the experiments in Fig. 2. The corresponding calculated transport characteristics are plotted in Fig. 3 for typical experimental parameters. Here, $-\epsilon_{i}$ corresponds to the changes in gate voltage (up to a lever arm factor). The model accounts for the shifts of the resonance maxima and minima and the peak-dip-peak transitions in $G_{2}$ with increasing magnetic field, as well as the relatively weak changes in $G_{1}$ at low fields.

To intuitively understand the structure of the conductance variations and the field evolution, we plot in Fig. 4(a) the local and CPS contributions to the total conductance $G_{2}$ for $B=0$ and $B=1.2 \mathrm{~T}$. We find that $G_{\mathrm{loc}}$ of both QDs often exhibits a strong asymmetric dip as a function of the respective far gate voltage, while $G_{\text {CPS }}$ generally results in a Lorentzian peak, not necessarily at the same position. The physical reason for these characteristics is the impact of interference: for the local processes into $N 1$, the lowest order nonzero contribution comprises two different paths. Either an electron tunnels directly from the central region to QD1 and to lead $N 1$, or it reaches QD1 and $N 1$ after an
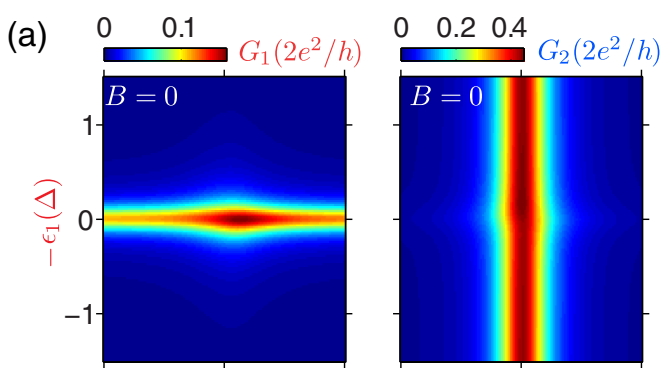

(b)
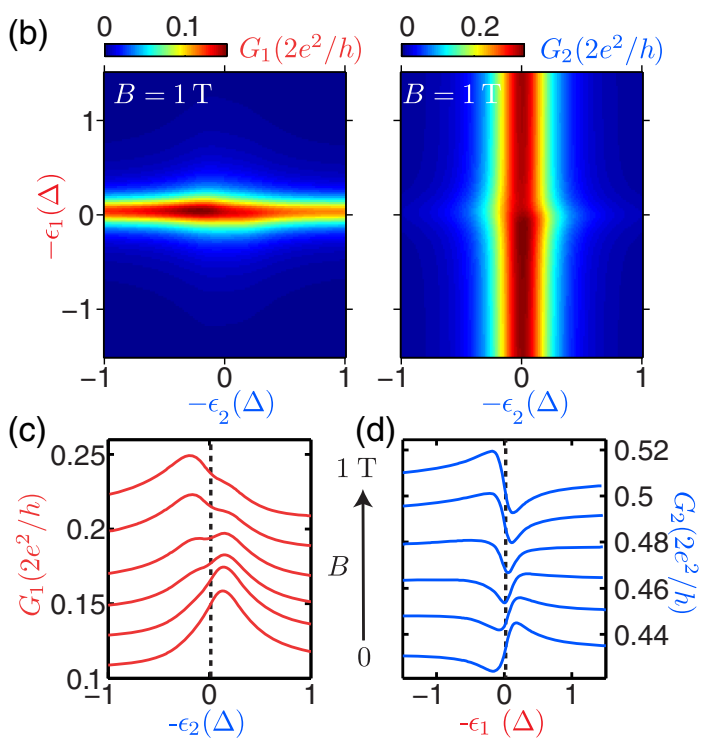

FIG. 3 (color online). Conductances through QD1 and QD2 in the three-site model with parameters chosen to qualitatively reproduce the experiments in Fig. 2. (a),(b) $G_{1}$ and $G_{2}$ as a function of both QD level positions. (c),(d) On-resonance conductance variations of QD1 and QD2, respectively, as a function of the level position of the other QD, for increasing values of the magnetic field. The parameters used in the calculations are $\epsilon_{m}=-0.03 \Delta, 2 t_{1 m}=t_{2 m}=0.1 \Delta, U=3 \Delta$, and the coupling to the leads $\gamma_{1}=0.15 \Delta, \gamma_{2}=0.2 \Delta$, and $\gamma_{m}=0.11 \Delta$.

excursion to QD2 and tunneling through the middle region, see Fig. 1(b). Since the transmission phase acquired on a QD state can vary by a large fraction of $\pi$ [30], the two paths can interfere constructively or destructively, depending on the relative level positions, resulting in a minimum or a maximum in the local contribution and in an asymmetric conductance variation. Especially when all resonances are at the Fermi energy, the wave function of path 2 in Fig. 1(b) is shifted by $\sim 2 \times \pi / 2$ with respect to path 1 , which results in a dip in the local contributions. In contrast to the local currents, the lowest-order nonzero CPS contribution stems from the direct tunneling of one electron into each QD, which is not affected by interference [23]. The sum of the local and nonlocal processes [31] can then result in the observed Fano-type line shapes.

The inversion of the line shape symmetry with the magnetic field, shown in Fig. 4(a), can be understood as follows: when $\epsilon_{m}$ is below (above) the Fermi energy, the 

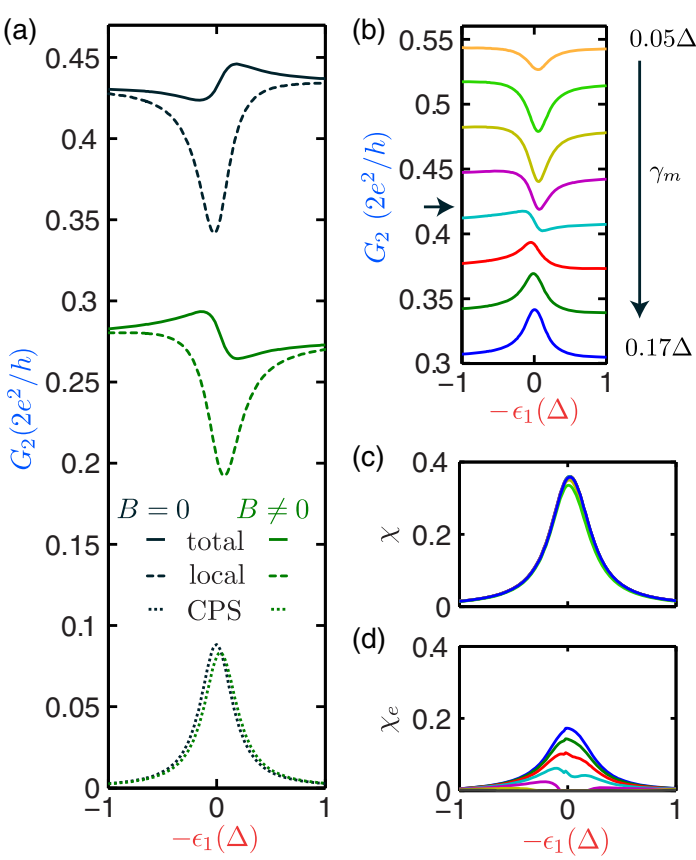

(c)

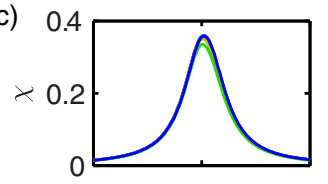

(d)

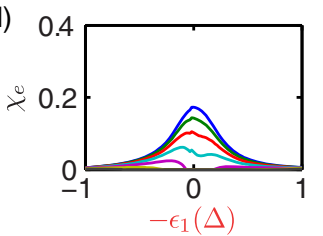

FIG. 4 (color online). (a) Total conductance through the QD2, $G_{2}$ (solid line), and the decomposition into the local (dashed line) and CPS (dotted line) contributions, as a function of $-\epsilon_{1}$ for $B=0$ (dark) and $B=1.2 \mathrm{~T}$ (green). (b) $G_{2}$ vs $V_{g 1}$ for different coupling strengths $\gamma_{m}$ to the superconductor. The curves are offset vertically for clarity. The value at which $\gamma_{m}=t_{i m}$ is pointed out by an arrow. (c),(d) Correct and estimated CPS efficiencies $\chi$ and $\chi_{e}$ for the numerical results in (b).

asymmetry of the local contribution results in a maximum in the total signal at a more positive (negative) gate voltage than the dip. In our model this symmetry is thus determined by $\epsilon_{m}$, which plays the same role as the shape parameter in the conventional Fano resonance [32].

In the experiments of Figs. 2(c) and 2(d) we find a transition from a peak-dip to a dip-peak structure at $B \approx 0.6 \mathrm{~T}$, which we thus identify as the field at which the position of the central level is shifted from below to above the Fermi energy by orbital and Zeeman shifts. We note that an asymmetric shape also shifts the maxima of the total conductance with respect to the resonance crossing. We observe these characteristics in the experiments [Figs. 2(c) and 2(d)] as well as in our calculations [Figs. 3(c) and 3(d)], where the maxima and minima do not occur at the dashed lines that indicate the crossings.

As can be inferred from Fig. 4(a), the CPS contribution does not change significantly with $B$ (for low enough $B$ ), but the local contribution can be reduced strongly. The latter is due to the lifting of the spin degeneracy by the Zeeman shift, which results in a reduction of LAR due to the opposite-spin electrons of a Cooper pair tunneling through a (partially) polarized level. This leads to an increase in the CPS efficiency defined as $\chi=2 G_{\mathrm{CPS}} /\left(G_{1}+G_{2}\right)[6,11]$.

The efficiency is relevant for most prospective applications of a CPS device $[11,17]$. In the above expression for $\chi$

one usually estimates $G_{\mathrm{CPS}}$ by subtracting the conductance sufficiently far away from the resonance crossing. We call this experimentally obtained efficiency $\chi_{e}$. It was shown for an incoherent model that this procedure underestimates the actual CPS efficiency [9]. However, this is not obvious for coherent transport. In Fig. 4(a) we show in an example that the local contribution is not constant, but exhibits a minimum at the resonance crossing, which also suggests that $\chi \geq \chi_{e}$. To further illustrate this effect, we have calculated $G_{2}$ vs $\epsilon_{1}$ (i.e., $V_{g 1}$ ) in the three-site model for a series of tunnel coupling strengths $\gamma_{m}$ between $S$ and the middle level, see Fig. 4(b). We find that the conductance minimum for weak couplings evolves into a maximum for $\gamma_{m} \gtrsim t_{i m}$ (black arrow). The reason for this transition is an additional contribution by states forming in the superconducting gap as $\gamma_{m}$ increases. In Fig. 4(c), $\chi$ is plotted for all curves in Fig. 4(b) and is almost identical for all $\gamma_{m}$. For comparison, $\chi_{e}$ is plotted in Fig. 4(d) for the same conductances, which demonstrates that $\chi_{e}$ systematically underestimates $\chi$ (we have omitted negative values) [33].

In summary, we report experiments on a Cooper pair splitting device that exhibits characteristics of quantum interference, which are tunable by electrical gates and external magnetic fields. To interpret these data, we introduce a three-site model that reproduces the experiments on a qualitative level and allows the decomposition of the conductances into local and CPS contributions. Specifically, we show that quantum interference is relevant in such structures, which is promising for any application of CPS as a source of entangled electron pairs. In addition, we discuss the impact of various device parameters and find that the experimentally estimated CPS efficiency is quite generally a lower bound for the actual value also in the coherent transport regime. The presented model might also be used to gain a new vantage point for other fundamental effects in superconducting proximity systems, such as fractional fermions or Majorana bound states. For example, a similar setup could be used to detect the inversion of the gap in a Majorana wire by the change of symmetry in the local Andreev conductance as a single level crosses the Fermi energy.

We gratefully acknowledge the financial support by the EU FP7 project SE ${ }^{2} \mathrm{ND}$, the EU ERC projects CooPairEnt and QUEST, the SCIEX project NoCoNano, the Swiss NCCR Quantum, the Swiss SNF, and the Danish Research Councils.

G. F, F. D., and S. dH. contributed equally to this work.

*andreas.baumgartner@unibas.ch

Present address: Danish Fundamental Metrology, DK-2800 Kongens Lyngby, Denmark.

[1] P. Recher, E. V. Sukhorukov, and D. Loss, Phys. Rev. B 63, 165314 (2001). 
[2] G. B. Lesovik, T. Martin, and G. Blatter, Eur. Phys. J. B 24, 287 (2001).

[3] O. Sauret, D. Feinberg, and T. Martin, Phys. Rev. B 70, 245313 (2004).

[4] V. Mourik, K. Zuo, S. M. Frolov, S. R. Plissard, E. P. A. M. Bakkers, and L. P. Kouwenhoven, Science 336, 1003 (2012).

[5] J. Nilsson, A. R. Akhmerov, and C. W. J. Beenakker, Phys. Rev. Lett. 101, 120403 (2008).

[6] L. Hofstetter, S. Csonka, J. Nygård, and C. Schönenberger, Nature (London) 461, 960 (2009).

[7] L. Hofstetter, S. Csonka, A. Baumgartner, G. Fülöp, S. d'Hollosy, J. Nygård, and C. Schönenberger, Phys. Rev. Lett. 107, 136801 (2011).

[8] A. Das, Y. Ronen, M. Heiblum, D. Mahalu, A. V. Kretinin, and H. Shtrikman, Nat. Commun. 3, 1165 (2012).

[9] G. Fülöp, S. d'Hollosy, A. Baumgartner, P. Makk, V. A. Guzenko, M. H. Madsen, J. Nygård, C. Schönenberger, and S. Csonka, Phys. Rev. B 90, 235412 (2014).

[10] L. G. Herrmann, F. Portier, P. Roche, A. L. Yeyati, T. Kontos, and C. Strunk, Phys. Rev. Lett. 104, 026801 (2010).

[11] J. Schindele, A. Baumgartner, and C. Schönenberger, Phys. Rev. Lett. 109, 157002 (2012).

[12] J. Schindele, A. Baumgartner, R. Maurand, M. Weiss, and C. Schönenberger, Phys. Rev. B 89, 045422 (2014).

[13] Z. B. Tan, D. Cox, T. Nieminen, P. Lähteenmäki, D. Golubev, G. B. Lesovik, and P. J. Hakonen, Phys. Rev. Lett. 114, 096602 (2015).

[14] G. Burkard, D. Loss, and E. V. Sukhorukov, Phys. Rev. B 61, R16303 (2000).

[15] S. Kawabata, J. Phys. Soc. Jpn. 70, 1210 (2001).

[16] B. Braunecker, P. Burset, and A. Levy Yeyati, Phys. Rev. Lett. 111, 136806 (2013).

[17] W. Klobus, A. Grudka, A. Baumgartner, D. Tomaszewski, C. Schönenberger, and J. Martinek, Phys. Rev. B 89, 125404 (2014).

[18] A. Cottet, Phys. Rev. B 86, 075107 (2012).

[19] Z. Scherübl, A. Palyi, and S. Csonka, Phys. Rev. B 89, 205439 (2014).
[20] M. H. Madsen, P. Krogstrup, E. Johnson, S. Venkatesan, E. Mühlbauer, C. Scheu, C. B. Sørensen, and J. Nygård, J. Cryst. Growth 364, 16 (2013).

[21] H. Shtrikman, R. Popovitz-Biro, A. Kretinin, L. Houben, M. Heiblum, M. Bukala, M. Galicka, R. Buczko, and P. Kacman, Nano Lett. 9, 1506 (2009).

[22] D. B. Suyatin, C. Thelander, M. T. Bjrk, I. Maximov, and L. Samuelson, Nanotechnology 18, 105307 (2007).

[23] See Supplemental Material at http://link.aps.org/ supplemental/10.1103/PhysRevLett.115.227003, which includes Ref. [24], for additional quantum dot characteristics, a detailed description of the theoretical model, a discussion of its limitations, and comments on the tunability of the central level.

[24] S. Datta, Electronic Transport in Mesoscopic Systems (Cambridge University Press, Cambridge, England, 1995).

[25] J. Rech, D. Chevallier, T. Jonckheere, and T. Martin, Phys. Rev. B 85, 035419 (2012).

[26] J. Eldridge, M. G. Pala, M. Governale, and J. König, Phys. Rev. B 82, 184507 (2010).

[27] S. Csonka, L. Hofstetter, F. Freitag, S. Oberholzer, T. S. Jespersen, M. Aagesen, J. Nygård, and C. Schönenberger, Nano Lett. 8, 3932 (2008).

[28] S. d'Hollosy, G. Fábián, A. Baumgartner, J. Nygård, and C. Schönenberger, AIP Conf. Proc. 1566, 359 (2013).

[29] P. Burset, W. J. Herrera, and A. L. Yeyati, Phys. Rev. B 84, 115448 (2011).

[30] R. Schuster, E. Buks, M. Heiblum, D. Mahalu, V. Umansky, and H. Shtrikman, Nature (London) 385, 417 (1997).

[31] The two contributions are added incoherently because of the different corresponding final states.

[32] U. Fano, Phys. Rev. 124, 1866 (1961).

[33] Clearly, $\chi_{e}$ overestimates $\chi$ for $\epsilon_{m} \rightarrow 0$ where the CPS contribution is masked by the global conductance dip, but we note that this also holds for $\epsilon_{m} \gg t_{i m}$ due to the misalignment of the peaks in the local contribution. 